\title{
Healthy side motor dysfunctions in facial paralysis
}

Marta Nichele

Muscular hyperactivity of facial muscles of the healthy side

exacerbating face asymmetry in absence of any other neurological lesion. 